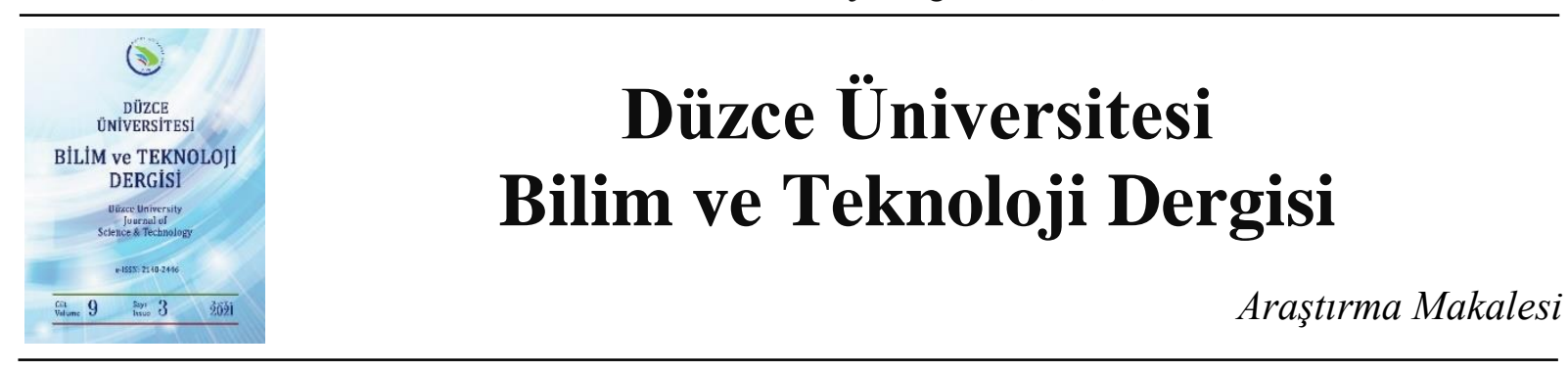

\title{
13 Atomlu Cu-Ag-Au Üçlü Nanoalaşımların Kimyasal Sıralama ve Yapısal Özelliklerinin İncelenmesi
}

\author{
Hüseyin YILDIRIM ${ }^{\mathrm{a}, *}$ \\ ${ }^{a}$ Yenice Meslek Yüksekokulu, Karabük Üniversitesi, Karabük, TÜRKIYE \\ * Sorumlu yazarin e-posta adresi: huseyinyildirim@karabuk.edu.tr \\ DOI: $10.29130 /$ dubited.845551
}

\begin{abstract}
ÖZET
$\mathrm{Bu}$ çalışmada, 13 atomlu $\mathrm{Cu}-\mathrm{Ag}$-Au üçlü metal nanoalaşımların kimyasal sıralama ve yapısal özellikleri Gupta ve DFT düzeylerinde ve üç farklı kompozisyon sisteminde incelenmiştir. $\mathrm{Cu}-\mathrm{Ag}-\mathrm{Au}$ üçlü nanoalaşımların Gupta düzeyindeki lokal optimizasyonları Basin-Hopping algoritması kullanılarak gerçekleştirilmiştir. Optimizasyon sonuçları Ag atomlarının yüzeye yerleşmeyi tercih ettiğini göstermektedir. $\mathrm{Cu}$ ve Au atomlarının nanoalaşımların yüzeyine veya merkezine ayrışma eğilimlerinin ise kompozisyon sistemine göre değiştiği bulunmuştur. $\mathrm{Cu}-\mathrm{Ag}$ Au nanoalaşımlarının tüm kompozisyonları için en kararlı kimyasal düzene sahip yapılar DFT relaksasyonu ile yeniden optimize edilmiştir ve Gupta ve DFT düzeylerinin karışma enerjileri karşılaştırılmıştır. Karışma enerjisi analizi, Gupta seviyesinde bulunan $\mathrm{Ag}_{1} \mathrm{Au}_{\mathrm{n}} \mathrm{Cu}_{12-\mathrm{n}}(\mathrm{n}=0-12)$ ve $\mathrm{Au}_{1} \mathrm{Ag}_{\mathrm{n}} \mathrm{Cu}_{12-\mathrm{n}}(\mathrm{n}=0-12)$ kompozisyon sistemlerinin en kararlı yapısının DFT ile uyuşmadığını göstermiştir.
\end{abstract}

Anahtar Kelimeler: Altın, Bakır, DFT, Gupta, Gümüş, Üçlü Nanoalaşım

\section{Investigation of The Chemical Ordering and Structural Properties of 13 Atom $\mathrm{Cu}-\mathrm{Ag}$-Au Ternary Nanoalloys}

\begin{abstract}
In this study, chemical ordering and structural properties of 13 -atom $\mathrm{Cu}$-Ag-Au ternary metal nanoalloys were investigated at Gupta and DFT levels and in three different composition systems. Local optimizations of $\mathrm{Cu}-\mathrm{Ag}$ Au ternary nanoalloys at Gupta level were performed by using Basin-Hopping algorithm. The optimization results show that Ag atoms prefer to locate to the surface. It is found that the tendency to segregation to the surface or centre of nanoalloys of $\mathrm{Cu}$ and $\mathrm{Au}$ atoms vary according to the composition system. The most stable chemical ordering structures for all compositions of $\mathrm{Cu}-\mathrm{Ag}$-Au nanoalloys have been re-optimized with DFT relaxations and the mixing energies of the Gupta and DFT levels are compared. The mixing energy analysis showed that the most stable structure of $\mathrm{Ag}_{1} \mathrm{Au}_{\mathrm{n}} \mathrm{Cu}_{12-\mathrm{n}}(\mathrm{n}=0-12)$ and $\mathrm{Au}_{1} \mathrm{Ag}_{\mathrm{n}} \mathrm{Cu}_{12-\mathrm{n}}(\mathrm{n}=0-12)$ composition systems found at Gupta level does not agree with those of DFT.
\end{abstract}

Keywords: Copper, DFT, Gold, Gupta, Silver, Ternary nanoalloy 


\section{GIRIS}

Son yıllarda, ikili ve üçlü nanoalaşımlar üzerine yapılan araştırmalar, metal nanoalaşımların kataliz, katı-hal fiziği, kimyasal fizik, biyotıp, optik, elektronik ve manyetik alanlardaki potansiyel uygulamaları nedeniyle önemli ilerlemeler kaydetmiştir [1,2]. Özellikle üçlü nanoalaşımlar daha karmaşık olmasına rağmen, yüksek katalitik aktivite ve seçiciliğe sahip olması nedeniyle ilgi çekmektedir [2]. Ayrıca, üçlü nanoalaşımlar nanokatalizörlerin aktivitesini iyileştirerek, daha düşük maliyetli ve isteğe bağl1 özelliklere sahip yeni işlevsel nanomalzemelerin tasarlanması için daha geniş olanaklar sunmaktadır [1, 3].

Grup 11 metalleri $(\mathrm{Cu}, \mathrm{Ag}, \mathrm{Au})$, çoğu katalizörün önemli bileşenleri olması ve bu metallerin elektronegatiflikleri, yüksek iletkenlikleri, kimyasal kararlılıkları, termal iletkenlikleri, düşük isıl genleşmeleri ve yüksek aktiviteleri nedeniyle modern katalizde büyük ilgi görmektedir. [4-7]. Bakır düşük maliyeti, bol rezervleri ve çok yönlülüğü nedeniyle kataliz olarak hidrojenasyon reaksiyonları ve dehidrojenasyonda yaygın olarak kullanılmaktadır [7, 8]. Gümüş, oksijen moleküllerinin aktivasyonu ve adsorpsiyon yeteneklerinden dolayı oksidasyon reaksiyonlarında en etkili katalizörlerden biri olarak kabul edilmektedir [8]. Altın ise katalizör olarak, oksidasyon, hidrojenasyon ve diğer birçok dönüşüm için başarı ile kullanılmaktadır [7]. Bu sonuçlar 1şığında, Grup 11 metalleri, oksidasyon aktivitelerinde önemli bir rol oynadığı için yakıt hücrelerindeki oksijen indirgeme reaksiyonu (ORR) için en iyi katalizörlerden biri olarak kabul edilmektedir [9-11]. Ayrıca, $\mathrm{Cu}-\mathrm{Ag}-\mathrm{Au}$ nanoalaşımları, yüzey ayrışmaları giderek daha fazla önem kazandığı için ve özellikleri iyi bilinen Grup 11 metallerinden oluştuğu için ilgi çekmektedirler [2, 12]. Bu nedenle, Grup 11 metallerinin kimyasal kökenini bulmak, katalitik davranışlarının anlaşılmasında önemli bir etkiye sahip olacağı ve gelecekteki uygulamalarının tasarımına rehberlik edeceği için, son yıllarda Grup 11 metalleri teorik olarak [13-19] oldukça fazla çalışılmıştır.

Rapallo ve arkadaşları, 34 ve 38 atomlu Ag-Cu nanoalaşımlarını optimize etmişlerdir ve optimizasyon sonucunda $\mathrm{Ag}-\mathrm{Cu}$ nanoalaşımlarında boyut uyumsuzluğu ve $\mathrm{Ag}$ atomlarının yüzeye yerleşme eğiliminde olduklarını bulmuşlardır [13]. Wilson ve Johnston, ikosahedron $\mathrm{Cu}-\mathrm{Au}$ nanoalaşımları için en düşük enerjili izomerleri araştırmışlardır ve Au atomlarının nanoalaşımın yüzey bölgesine, $\mathrm{Cu}$ atomlarının nanoalaşımın merkezine yerleşme eğiliminde olduklarını bulmuşlardır [14]. Wu ve arkadaşları, 55 atomlu Ag-Au nanoalaşımlarını Gupta düzeyinde optimize etmişlerdir ve optimizasyon sonucunda nanoalaşımların hepsinin İkosahedron yapıya sahip olmadığını ve yapısal olarak üç sınıfa ayrıldığını bulmuşlardır [15]. Wu ve arkadaşları 19 ve 55 atomdan oluşan $\mathrm{Cu}-\mathrm{Ag}$ - $\mathrm{Au}$ üçlü nanoalaşımların optimizasyonunu içeren çalışmalarında, 19 atomdan oluşan $\mathrm{Cu}-\mathrm{Ag}-\mathrm{Au}$ üçlü nanoalaşımların optimizasyonu sonrasında bütün nanoalaşımların iç içe geçmiş ikili ikosahedron yapıya sahip olduğunu ve 55 atomdan oluşan $\mathrm{Cu}-\mathrm{Ag}$ - $\mathrm{Au}$ nanoalaşımların ise optimizasyondan sonra $\mathrm{Cu}$ ve $\mathrm{Ag}$ atomlarının nanoalaşımın merkezine ve yüzeye, Au atomlarının çoğunlukla ikinci kabuğa yerleştiğini bulmuşlardır [2]. Cheng ve arkadaşları $\mathrm{Ag}-\mathrm{Cu}-\mathrm{Au}$ üçlü nanoalaşımların yüzey ayrışmalarını incelemişlerdir ve Ag atomlarının genellikle nanoalaşımların yüzeyinde toplandığını, Au atomlarının nanoalaşımın iç kabuklarına yerleştiğini ve $\mathrm{Cu}$ atomlarının ise nanoalaşımın merkezinde yer aldığını bulmuşlardır [12]. Yıldırım ve Arslan iki ayrı çalışmada 23, 26, 38 ve 55 atomlu $\mathrm{Cu}-\mathrm{Ag}-\mathrm{Au}$ nanoalaşımlarının yapısal özelliklerini her büyüklükteki nanoalaşımlarda sadece $\mathrm{Cu}$ atom sayısını sabit tutarak Gupta düzeyinde incelemiş̧lerdir. Optimizasyonlar sonucunda $\mathrm{Ag}$ ve $\mathrm{Au}$ atomlarının yüzeylere, $\mathrm{Cu}$ atomunun ise genellikle iç kabuklara yerleşme eğiliminde olduklarını bulmuşlardır $[16,17]$. Goh ve arkadaşları $\mathrm{AuCu}$ nanoalaşımları için yeni bir Gupta potansiyel parametre seti önermişlerdir ve sonuçlarını Yoğunluk Fonksiyonel Teorisi (Density Functional Theory, DFT) hesaplamalarıla karşılaştırmışlardır. Önerilen parametre setinde elde edilen kimyasal sıralama sonuçlarının DFT hesaplamaları ile elde edilen sonuçlar ile çok uyumlu olduğunu bulmuşlardır. [18]. Jiang ve arkadaşları DFT düzeyinde küçük $\mathrm{Cu}_{\mathrm{n}-1} \mathrm{Ag}(2 \leq \mathrm{n} \leq 8)$ nanoalaşımları için Ag'nin yüzey ayrışma eğilimini incelemişlerdir ve Ag atomlarının yüzey pozisyonlarını işgal etme eğiliminde olduklarını bulmuşlardır [19]. 
Nanoalaşımların kimyasal düzeni ve geometrik şekli, nanoalaşımların özelliklerinin belirlenmesinde, kullanışlı ve pahalı olmayan katalitik malzeme tasarlanmasında önemli rol oynamaktadır [20, 21]. Özellikle, çekirdek kabuk yapısına sahip nanoalaşımlar yüksek simetriye sahip olmaları ve çekirdek ve kabuk atomları arasındaki yüzey geriliminin katalitik aktiviteyi etkilemesinden dolayı ilgi çekmektedirler [17, 20, 22, 23]. Bu çalışmada 13 atomlu ikosahedron geometriye yoğunlaşmamızın sebebi, ikosahedron geometrinin farklı geometriler arasında en yüksek simetriye sahip olması ve 13'ün ikosahedron geometri için sihirli bir sayı ve çekirdek-kabuk yapısına sahip olmasıdır [17, 21, 24].

$\mathrm{Bu}$ çalışmada, 13 atomlu $\mathrm{Cu}-\mathrm{Ag}$-Au nanoalaşımların yapısal özellikleri hakkında yeterli literatür bilgisi olmadığı için, $\mathrm{Cu}-\mathrm{Ag}-\mathrm{Au}$ nanoalaşımları üç farklı kompozisyon sistemi ile ele alınarak incelenmiştir. Üç farklı sistemi oluşturan $\mathrm{Cu}_{1} \mathrm{Ag}_{\mathrm{n}} \mathrm{Au}_{12-\mathrm{n}}(\mathrm{n}=0-12), \mathrm{Ag}_{1} \mathrm{Au}_{\mathrm{n}} \mathrm{Cu}_{12-\mathrm{n}}(\mathrm{n}=0-12)$ ve $\mathrm{Au}_{1} \mathrm{Ag}_{\mathrm{n}} \mathrm{Cu}_{12-\mathrm{n}}(\mathrm{n}=0-12)$ nanoalaşımlarının bir tane atomu sabit alınarak ve diğer iki atomun toplam sayısı 12 olacak şekilde lokal optimizasyonları gerçekleştirilmiştir. 13 atomdan oluşan $\mathrm{Cu}-\mathrm{Ag}-\mathrm{Au}$ nanoalaşımlarının Gupta düzeyindeki lokal optimizasyonları sonucunda bulunan en kararlı kimyasal düzene sahip yapılar daha sonra DFT relaksasyonu ile yeniden optimize edilmiştir ve Gupta ve DFT düzeylerinin karışma enerjileri karşıllaştırılmıştır. DFT, atomlarla ilgili modellerin doğruluğunu değerlendirmeye izin verdiğinden, nanoalaşımların yapısal davranışlarını keşfetmek için en popüler yaklaşımlardan biridir ve çoğu durumda Gupta potansiyeli ile oldukça iyi bir uyum elde etmektedir [1,3].

\section{HESAPLAMA YÖNTEMI}

$\mathrm{Bu}$ çalışmada $\mathrm{Cu}_{1} \mathrm{Ag}_{\mathrm{n}} \mathrm{Au}_{12-\mathrm{n}}$ ( $\left.\mathrm{n}=0-12\right), \mathrm{Ag}_{1} \mathrm{Au}_{\mathrm{n}} \mathrm{Cu}_{12-\mathrm{n}} \quad(\mathrm{n}=0-12)$ ve $\mathrm{Au}_{1} \mathrm{Ag}_{\mathrm{n}} \mathrm{Cu}_{12-\mathrm{n}} \quad(\mathrm{n}=0-12)$ üçlü nanoalaşımların yapısal analizleri atomistik ve DFT hesaplamalarıla yapılmıştır. Yapısal araştırmalarımız için ilk olarak $\mathrm{Cu}_{1} \mathrm{Ag}_{\mathrm{n}} \mathrm{Au}_{12-\mathrm{n}}(\mathrm{n}=0-12), \mathrm{Ag}_{1} \mathrm{Au}_{\mathrm{n}} \mathrm{Cu}_{12-\mathrm{n}}(\mathrm{n}=0-12)$ ve $\mathrm{Au}_{1} \mathrm{Ag}_{\mathrm{n}} \mathrm{Cu}_{12-\mathrm{n}}(\mathrm{n}=0-$ 12) üçlü nanoalaşımların en kararlı kimyasal düzene sahip yapıları Basin-Hopping algoritması kullanılarak GMIN programı ile elde edilmiştir [25]. Her nanoalaşım için Basin-Hopping algoritması $2 \times 10^{5}$ adımda gerçekleştirilmiştir. 13 atomlu $\mathrm{Cu}-\mathrm{Ag}-\mathrm{Au}$ nanoalaşımlarında $\mathrm{Cu}, \mathrm{Ag}$ ve $\mathrm{Au}$ atomları arasındaki etkileşmeler Gupta çok cisim potansiyel enerji fonksiyonu ile modellenmiştir [26]. Gupta çok cisim potansiyel enerji fonksiyonu, $V_{i}^{r}$ itici çift bileşen ve $V_{i}^{m}$ çekici çok cisim bileşen terimlerinin toplamidir [27-29].

$$
\begin{aligned}
& V=\sum_{i}^{N}\left(V_{i}^{r}-V_{i}^{m}\right) \\
& V_{i}^{r}=\sum_{j \neq i}^{N} A(a, b) \exp \left(-p(a, b)\left(\frac{r_{i j}-r_{0}(a, b)}{r_{0}(a, b)}\right)\right) \\
& V_{i}^{m}=\sum_{j \neq i}^{N} \xi^{2}(a, b) \exp \left[-2 q(a, b)\left(\frac{r_{i j}-r_{0}(a, b)}{r_{0}(a, b)}\right)\right]^{2}
\end{aligned}
$$

Gupta potansiyelinde $a$ ve $b, i$ ve $j$ atomlarının türlerini, $r_{i j}, i$. ve $j$. atomlar arası uzaklığ 1 ve $r_{0}$ en yakın komşu mesafesini ifade eden parametrelerdir. $A, p, q$ ve $\xi$ parametreleri kohesif enerji, örgü parametreleri ve bağımsız elastik sabitlerin deneysel değerlerine mutlak sıfırdaki referans bulk kristal yapı için fit edilmiştir. Bu çalışmada kullanılan Gupta parametreleri Tablo 1'de verilmiştir [2].

Lokal optimizasyonlar sonucunda elde edilen en kararlı kimyasal düzene sahip yapılar DFT relaksasyonu için başlangıç konfigürasyonları olarak kullanılmıştır. DFT hesaplamaları ise, Quantum Espresso'da (QE) PWscf kodu [30, 31], pseudopotansiyeller [32] ve Perdew Burke Ernzerhof (PBE) tarafından önerilen genelleştirilmiş gradyent yaklaşımı (GGA) kullanarak yapılmıştır [33]. Dalga fonksiyonu enerji kesim değeri 41.0 Ry ve yük yoğunluğu enerji kesim değeri 364.0 Ry olarak 
seçilmiştir. Marzari-Vanderbilt smearing tekniği 0.02 Ry genişletilerek uygulanmıştır. Tüm DFT hesaplamalarında küme bir kenarı 17 Å olan kübik bir hücrenin merkezine yerleştirilmiştir.

Tablo 1. Gupta potansiyel enerji fonksiyonu parametreleri.

\begin{tabular}{cccccc}
\hline & $\begin{array}{c}\mathbf{A} \\
(\mathrm{eV})\end{array}$ & $\mathbf{p}$ & $\mathbf{q}$ & $\begin{array}{c}\boldsymbol{\xi} \\
(\mathrm{eV})\end{array}$ & $\begin{array}{c}\mathbf{r}_{\mathbf{0}} \\
(\AA)\end{array}$ \\
\hline $\mathrm{Cu}-\mathrm{Cu}$ & 0.0855 & 10.960 & 2.2780 & 1.2240 & 2.5562 \\
$\mathrm{Cu}-\mathrm{Ag}$ & 0.0980 & 10.700 & 2.8050 & 1.2274 & 2.7224 \\
$\mathrm{Cu}-\mathrm{Au}$ & 0.1539 & 11.050 & 3.0475 & 1.5605 & 2.5562 \\
$\mathrm{Ag}-\mathrm{Ag}$ & 0.1028 & 10.928 & 3.1390 & 1.1780 & 2.8885 \\
$\mathrm{Ag}-\mathrm{Au}$ & 0.1490 & 10.494 & 3.6070 & 1.4874 & 2.8864 \\
$\mathrm{Au}-\mathrm{Au}$ & 0.2061 & 10.229 & 4.0360 & 1.7900 & 2.8843 \\
\hline
\end{tabular}

\section{BULGULAR ve TARTISMA}

$\mathrm{Bu}$ çalışmada, 13 atomlu $\mathrm{Cu}-\mathrm{Ag}$ - $\mathrm{Au}$ nanoalaşımlarının yapısal özelliklerini incelemek için $\mathrm{Cu}_{1} \mathrm{Ag}_{\mathrm{n}} \mathrm{Au}_{12-}$ $\mathrm{n}(\mathrm{n}=0-12), \mathrm{Ag}_{1} \mathrm{Au}_{\mathrm{n}} \mathrm{Cu}_{12-\mathrm{n}}(\mathrm{n}=0-12)$ ve $\mathrm{Au}_{1} \mathrm{Ag}_{\mathrm{n}} \mathrm{Cu}_{12-\mathrm{n}}(\mathrm{n}=0-12)$ gibi üç farklı kompozisyon sistemi ele alınmıştır. Bu kompozisyon sistemlerinde yer alan nanoalaşımların lokal optimizasyonları BasinHopping algoritması kullanılarak gerçekleştirilmiştir. Gupta düzeyindeki optimizasyonlar sonucunda üç farklı sistemde bulunan bütün nanoalaşımların en kararlı kimyasal düzene sahip yapılarının OVITO'daki [34] Ortak Komşu Analizine göre ikosahedron yapıdadır. Ortak Komşu Analizi (Common Neighbour Analysis), FCC, HCP, BCC ve ikosahedron gibi farklı geometrik yapıları ayırt etmek için kullanılan faydalı bir araçtır [35]. 13 atomlu ikosahedron geometrinin bir atomu merkezde diğer 12 atomu ise yüzeyde bulunmaktadır. $\mathrm{Cu}_{1} \mathrm{Ag}_{\mathrm{n}} \mathrm{Au}_{12-\mathrm{n}}(\mathrm{n}=0-12)$ nanoalaşımlarının lokal optimizasyonları sonucunda bulunan Gupta düzeyindeki en düşük enerjiye ve en kararlı kimyasal düzene sahip yapıları Şekil 1'de gösterilmiştir. Şekil 1'de $\mathrm{Cu}, \mathrm{Ag}$ ve $\mathrm{Au}$ atomları sırasıyla kırmızı, gri ve sarı renkli küreler ile gösterilmiştir.

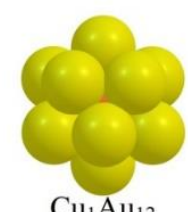

$\mathrm{Cu}_{1} \mathrm{Au}_{12}$

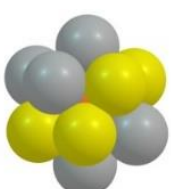

$\mathrm{Cu}_{1} \mathrm{Ag}_{6} \mathrm{Au}_{6}$

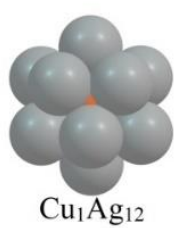

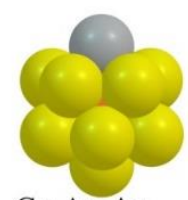

$\mathrm{Cu}_{1} \mathrm{Ag}_{1} \mathrm{Au}_{11}$

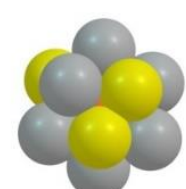

$\mathrm{Cu}_{1} \mathrm{Ag}_{7} \mathrm{Au}_{5}$

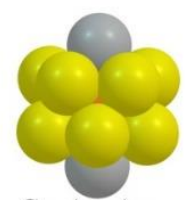

$\mathrm{Cu}_{1} \mathrm{Ag}_{2} \mathrm{Au}_{10}$

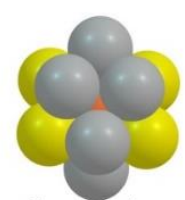

$\mathrm{Cu}_{1} \mathrm{Ag}_{8} \mathrm{Au}_{4}$

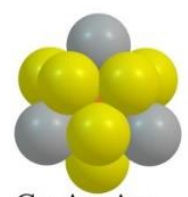

$\mathrm{Cu}_{1} \mathrm{Ag}_{3} \mathrm{Au} 9$

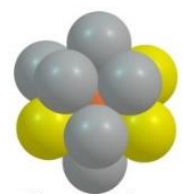

$\mathrm{Cu}_{1} \mathrm{Ag}_{9} \mathrm{Au}_{3}$

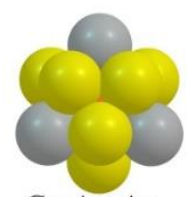

$\mathrm{Cu}_{1} \mathrm{Ag}_{4} \mathrm{Au}_{8}$

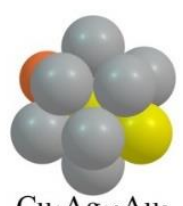

$\mathrm{Cu}_{1} \mathrm{Ag}_{10} \mathrm{Au}_{2}$

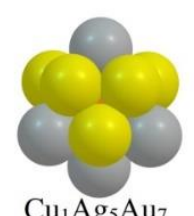

$\mathrm{Cu}_{1} \mathrm{Ag}_{5} \mathrm{Au}_{7}$

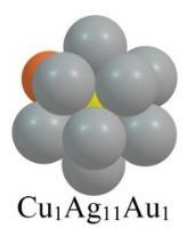

Sekil 1. $\mathrm{Cu}_{1} A g_{n} A u_{12-n}(n=0-12)$ nanoalașımlarının lokal optimizasyonları sonucunda elde edilen en kararlı kimyasal düzene sahip yapılar.

Şekil 1'e göre, $\mathrm{Cu}_{1} \mathrm{Au}_{12}$ ve $\mathrm{Cu}_{1} \mathrm{Ag}_{12}$ ikili nanoalaşımlarında 1 tane $\mathrm{Cu}$ atomunun nanoalaşımın merkezine, sırasıyla $12 \mathrm{Au}$ ve $12 \mathrm{Ag}$ atomunun ise yüzeye yerleştiği görülmektedir. $\mathrm{Cu}_{1} \mathrm{Au}_{12}$ nanoalaşımından, $\mathrm{Cu}_{1} \mathrm{Ag}_{9} \mathrm{Au}_{3}$ nanoalaşımına kadar ikosahedron yapının merkezine $\mathrm{Cu}$ atomunun 
yerleştiği görülmektedir. $\mathrm{Cu}_{1} \mathrm{Ag}_{10} \mathrm{Au}_{2}$ ve $\mathrm{Cu}_{1} \mathrm{Ag}_{11} \mathrm{Au}_{1}$ nanoalaşımlarında ise merkezdeki $1 \mathrm{Cu}$ atomu ile yüzeydeki $1 \mathrm{Au}$ atomu yer değiştirerek, $1 \mathrm{Au}$ atomu merkeze, $1 \mathrm{Cu}$ atomu ise yüzeye yerleşmektedir.

$\mathrm{Ag}_{1} \mathrm{Au}_{\mathrm{n}} \mathrm{Cu}_{12-\mathrm{n}}(\mathrm{n}=0-12)$ nanoalaşımlarının lokal optimizasyonları sonucunda bulunan Gupta düzeyindeki en düşük enerjiye ve en kararlı kimyasal düzene sahip yapıları Şekil 2'de gösterilmiştir. Şekil 2'e göre $\mathrm{Ag}_{1} \mathrm{Cu}_{12}$ ikili nanoalaşımında $1 \mathrm{Ag}$ atomunun nanoalaşımın yüzeyine yerleştiği görülmektedir. Nanoalaşımın merkezine ise $\mathrm{Cu}$ atomu yerleşmiştir. $\mathrm{Ag}_{1} \mathrm{Au}_{1} \mathrm{Cu}_{11}$ nanoalaşımından $\mathrm{Ag}_{1} \mathrm{Au}_{3} \mathrm{Cu}_{9}$ nanoalaşımına kadar nanoalaşımın merkezine $\mathrm{Au}$ atomu yerleşirken, $\mathrm{Cu}$ ve $\mathrm{Ag}$ atomları nanoalaşımın yüzeyine yerleşmektedir. $\mathrm{Ag}_{1} \mathrm{Au}_{4} \mathrm{Cu}_{8}$ nanoalaşımından $\mathrm{Ag}_{1} \mathrm{Au}_{11} \mathrm{Cu}_{1}$ nanoalaşımına kadar nanoalaşımın merkezine $\mathrm{Cu}$ atomu yerleşirken, yüzeydeki diğer $\mathrm{Cu}$ atomları ile $\mathrm{Au}$ atomları yer değiştirmektedir. $\mathrm{Bu}$ nanoalaşımlarda $1 \mathrm{Ag}$ atomu daima nanoalaşımın yüzeyine yerleşmektedir. $\mathrm{Ag}_{1} \mathrm{Au}_{12}$ ikili nanoalaşımında ise $1 \mathrm{Ag}$ atomu nanoalaşımın yüzeyine yerleşirken, nanoalaşımın merkezine $\mathrm{Au}$ atomunun yerleştiği görülmektedir.

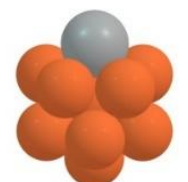

$\mathrm{Ag}_{1} \mathrm{Cu}_{12}$

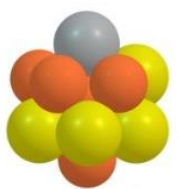

$\mathrm{Ag}_{1} \mathrm{Au}_{6} \mathrm{Cu}_{6}$

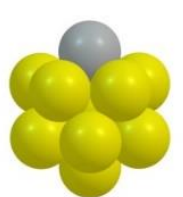

$\mathrm{Ag}_{1} \mathrm{Au}_{12}$

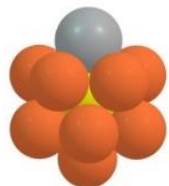

$\mathrm{Ag}_{1} \mathrm{Au}_{1} \mathrm{Cu}_{11}$

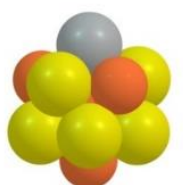

$\mathrm{Ag}_{1} \mathrm{Au}_{7} \mathrm{Cu}_{5}$

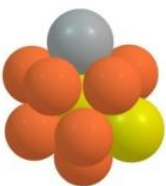

$\mathrm{Ag}_{1} \mathrm{Au}_{2} \mathrm{Cu}_{10}$

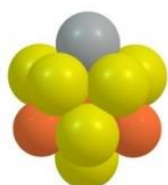

$\mathrm{Ag}_{1} \mathrm{Au}_{8} \mathrm{Cu}_{4}$

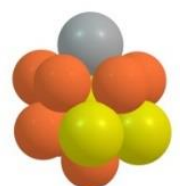

$\mathrm{Ag}_{1} \mathrm{Au}_{3} \mathrm{Cu}_{9}$

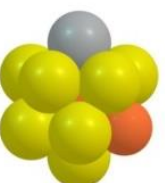

$\mathrm{Ag}_{1} \mathrm{Au}_{9} \mathrm{Cu}_{3}$

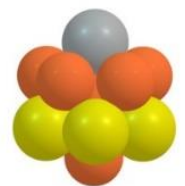

$\mathrm{Ag}_{1} \mathrm{Au}_{4} \mathrm{Cu}_{8}$

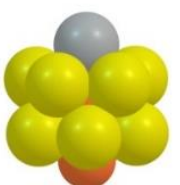

$\mathrm{Ag}_{1} \mathrm{Au}_{10} \mathrm{Cu}_{2}$

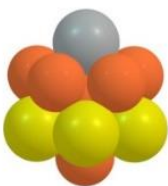

$\mathrm{Ag}_{1} \mathrm{Au}_{5} \mathrm{Cu}_{7}$

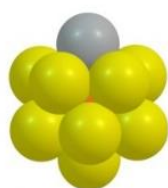

$\mathrm{Ag}_{1} \mathrm{Au}_{11} \mathrm{Cu}_{1}$

Şekil 2. $A g_{1} A u_{n} C u_{12-n}(n=0-12)$ nanoalaşımlarının lokal optimizasyonlart sonucunda elde edilen en kararlı kimyasal düzene sahip yapılar.

$\mathrm{Au}_{1} \mathrm{Ag}_{\mathrm{n}} \mathrm{Cu}_{12-\mathrm{n}}(\mathrm{n}=0-12)$ nanoalaşımlarının lokal optimizasyonları sonucunda bulunan Gupta düzeyindeki en düşük enerjiye ve en kararlı kimyasal düzene sahip yapıları Şekil 3'te gösterilmiştir. Şekil 3'e göre, bu nanoalaşımların hepsinde $1 \mathrm{Au}$ atomu daima nanoalaşımın merkezine yerleşirken, nanoalaşımdaki $\mathrm{Ag}$ atomu sayısı arttıkça, yüzeydeki $\mathrm{Ag}$ ve $\mathrm{Cu}$ atomları yer değiştirmektedir.

Tablo 2. Ag, Au, Cu elementlerinin özellikleri.

\begin{tabular}{lll}
\hline & $\begin{array}{l}\mathbf{E}_{\text {yüzey }} \\
\left(\mathrm{meVA}^{-2}\right)\end{array}$ & $\begin{array}{l}\mathbf{r}_{\text {atomik yarçap }} \\
(\AA)\end{array}$ \\
\hline $\mathrm{Ag}$ & 78.0 & 1.45 \\
$\mathrm{Au}$ & 96.8 & 1.44 \\
$\mathrm{Cu}$ & 113.9 & 1.28 \\
\hline
\end{tabular}

Sonuç olarak, Gupta düzeyinde $\mathrm{Cu}_{1} \mathrm{Ag}_{\mathrm{n}} \mathrm{Au}_{12-\mathrm{n}}(\mathrm{n}=0-12)$ ve $\mathrm{Ag}_{1} \mathrm{Au}_{\mathrm{n}} \mathrm{Cu}_{12-\mathrm{n}}(\mathrm{n}=0-12)$ nanoalaşımlarında $\mathrm{Cu}, \mathrm{Ag}$ ve $\mathrm{Au}$ atomlarının merkeze veya yüzeye yerleşme eğilimlerinin birbirine benzediği görülmüştür. $\mathrm{Cu}$ atomları genellikle merkeze yerleşmeyi tercih ederken, $\mathrm{Ag}$ ve $\mathrm{Au}$ atomları ise yüzeye yerleşmeyi tercih etmektedirler. $\mathrm{Cu}, \mathrm{Ag}$ ve $\mathrm{Au}$ atomlarının merkeze veya yüzeye yerleşme eğilimi atomların yüzey enerjilerine ve atomik yarıçaplarına bağlı olarak açıklanabilmektedir. Nanoalaşımlarda diğer atomlardan daha büyük yüzey enerjisine ve daha küçük atomik yarıçapa sahip atomlar nanoalaşımın merkezine yerleşmektedirler. $\mathrm{Cu}$ atomu Tablo 2'de [36] verildiği üzere, diğer atomlardan daha büyük yüzey 
enerjisine ve daha düşük atomik yarıçapa sahip olduğu için bu iki kompozisyon sisteminde genellikle merkeze yerleşmiştir. $\mathrm{Au}_{1} \mathrm{Ag}_{\mathrm{n}} \mathrm{Cu}_{12-\mathrm{n}} \quad(\mathrm{n}=0-12)$ nanoalaşımlarında ise $\mathrm{Cu}_{1} \mathrm{Ag}_{\mathrm{n}} \mathrm{Au}_{12-\mathrm{n}} \quad(\mathrm{n}=0-12)$ ve $\mathrm{Ag}_{1} \mathrm{Au}_{\mathrm{n}} \mathrm{Cu}_{12-\mathrm{n}}(\mathrm{n}=0-12)$ nanoalaşımlarına göre $\mathrm{Cu}, \mathrm{Ag}$ ve $\mathrm{Au}$ atomlarının farklı yerleşme eğilimine sahip olduğu görülmüş̧ür. $\mathrm{Bu}$ kompozisyon sisteminde $\mathrm{Au}$ atomu bütün nanoalaşımlarda merkeze yerleşmektedir. $\mathrm{Cu}_{1} \mathrm{Ag}_{\mathrm{n}} \mathrm{Au}_{12-\mathrm{n}}(\mathrm{n}=0-12)$ ve $\mathrm{Ag}_{1} \mathrm{Au}_{\mathrm{n}} \mathrm{Cu}_{12-\mathrm{n}}(\mathrm{n}=0-12)$ kompozisyon sistemleri, yüzey enerjisi ve atomik yarıçap değerlerine göre öne sürülen $\mathrm{Cu}_{\text {merkez }}(\mathrm{AgAu})_{\text {yüzey }}$ yerleşimlerini desteklerken, $\mathrm{Au}_{1} \mathrm{Ag}_{\mathrm{n}} \mathrm{Cu}_{12-\mathrm{n}}(\mathrm{n}=0-12)$ kompozisyon sistemi desteklememektedir. $\mathrm{Cu}, \mathrm{Ag}$ ve $\mathrm{Au}$ atomlarının Gupta düzeyinde nanoalaşımdaki yapısal davranışlarında kompozisyon sisteminin etkisi olduğu görülmektedir.

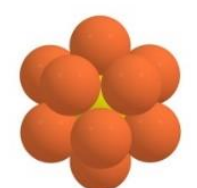

$\mathrm{Au}_{1} \mathrm{Cu}_{12}$

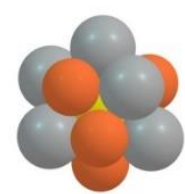

$\mathrm{Au}_{1} \mathrm{Ag}_{6} \mathrm{Cu}_{6}$

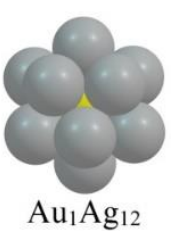

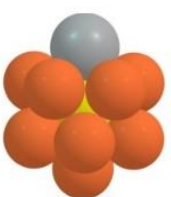

$\mathrm{Au}_{1} \mathrm{Ag}_{1} \mathrm{Cu}_{11}$

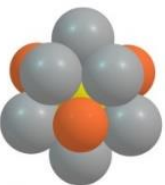

$\mathrm{Au}_{1} \mathrm{Ag}_{7} \mathrm{Cu}_{5}$

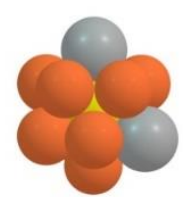

$\mathrm{Au}_{1} \mathrm{Ag}_{2} \mathrm{Cu}_{10}$

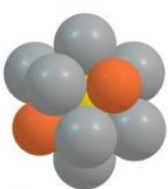

$\mathrm{Au}_{1} \mathrm{Ag}_{8} \mathrm{Cu}_{4}$

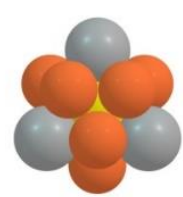

$\mathrm{Au}_{1} \mathrm{Ag}_{3} \mathrm{Cu}_{9}$

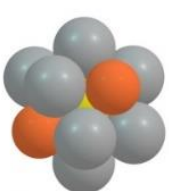

$\mathrm{Au}_{1} \mathrm{Ag}_{9} \mathrm{Cu}_{3}$

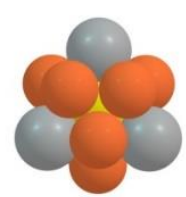

$\mathrm{Au}_{1} \mathrm{Ag}_{4} \mathrm{Cu}_{8}$

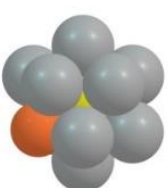

$\mathrm{Au}_{1} \mathrm{Ag}_{10} \mathrm{Cu}_{2}$

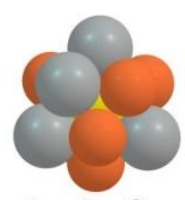

$\mathrm{Au}_{1} \mathrm{Ag}_{5} \mathrm{Cu}_{7}$

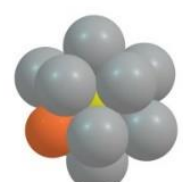

$\mathrm{Au}_{1} \mathrm{Ag}_{11} \mathrm{Cu}_{1}$

Şekil 3. $\mathrm{Au}_{1} \mathrm{Ag}_{n} \mathrm{Cu}_{12-n}(n=0-12)$ nanoalaşımlarının lokal optimizasyonları sonucunda elde edilen en kararlı kimyasal düzene sahip yapılar.

Nanoalaşımların yapısal olarak incelenmesi için önemli analiz yöntemlerinden biri de nanoalaşımların kompozisyona göre kararlılı̆̆ını araştırmak için en kararlı kimyasal düzene sahip yapıların enerjilerini kullanan karışma enerjisi $\left(\mathrm{E}_{\mathrm{exc}}\right)$ analizidir. Nanoalaşımda sayısı sabitlenen atom türü varsa, lokal optimizasyonlar sonucunda elde edilen üçlü nanoalaşımlar için karışma enerjisi Eşitlik (4) ile tanımlanmaktadır [17].

$\Delta E_{\text {exc }}^{\text {Gupta }}\left(C_{k} A_{n} B_{m}\right)=E\left(C_{k} A_{n} B_{m}\right)-n \frac{E\left(C_{k} A_{n+m}\right)}{n+m}-m \frac{E\left(C_{k} B_{n+m}\right)}{n+m}$

Burada, $E\left(C_{k} A_{n} B_{m}\right)$ üçlü nanoalaşımlarının toplam Gupta enerjisini, $E\left(C_{k} A_{n+m}\right)$ ve $E\left(C_{k} B_{n+m}\right)$ ise ikili nanoalaşımların toplam Gupta enerjilerini temsil etmektedir. Bu eşitlik $\Delta E_{\text {exc }}^{D F T}$ için kullanıldığında $E\left(C_{k} A_{n} B_{m}\right), E\left(C_{k} A_{n+m}\right)$ ve $E\left(C_{k} B_{n+m}\right)$ DFT toplam enerjileri ifade etmektedir [37]. Nanoalaşımlar için uyarlanan karışma enerjisi, nanoalaşımı oluşturan metallerin karışma derecesini vermektedir. $\mathrm{Bu}$ derecenin en düşük değeri, karışımın en uygun olduğu ve dolayısıyla en kararlı nanoalaşımı temsil etmektedir.

Cu-Ag-Au nanoalaşımlarının Gupta ve DFT düzeylerindeki E exc eğrileri Şekil 4, Şekil 5 ve Şekil 6'da verilmiştir.

$\mathrm{Ag}$ atomlarının sayısının (n) bir fonksiyonu olarak $\mathrm{Cu}_{1} \mathrm{Ag}_{\mathrm{n}} \mathrm{Au}_{12-\mathrm{n}}(\mathrm{n}=0-12)$ nanoalaşımlarının karışma enerjisi grafiği Şekil 4'te verilmiştir. Şekil 4'e göre, $\mathrm{Cu}_{1} \mathrm{Ag}_{\mathrm{n}} \mathrm{Au}_{12-\mathrm{n}}(\mathrm{n}=0-12)$ nanoalaşımlarının hem Gupta düzeyinde hem de DFT düzeyinde en kararlı nanoalaşımı $\mathrm{Cu}_{1} \mathrm{Ag}_{6} \mathrm{Au}_{6}$ olarak elde edilmiştir. $\mathrm{Bu}$ sonuç $\mathrm{Cu}_{1} \mathrm{Ag}_{\mathrm{n}} \mathrm{Au}_{12-\mathrm{n}}(\mathrm{n}=0-12)$ kompozisyon sistemi için Gupta ve DFT düzeylerinin birbiriyle uyumlu olduğunu göstermektedir. $\mathrm{Cu}_{1} \mathrm{Ag}_{6} \mathrm{Au}_{6}$ nanoalaşımının Ortak Komşu Analizine göre hem Gupta 
düzeyinde hem de DFT düzeyinde ikosahedron geometriye sahip olduğu görülmüştür. Ayrıca hem Gupta düzeyindeki hem de DFT düzeyindeki $\mathrm{Cu}_{1} \mathrm{Ag}_{6} \mathrm{Au}_{6}$ nanoalaşımında $1 \mathrm{Cu}$ atomu nanoalaşımın merkezine yerleşirken, $6 \mathrm{Ag}$ ve $6 \mathrm{Au}$ atomu nanoalaşımın yüzeyine birbirleriyle karışarak yerleşmiştir. $\mathrm{Cu}_{1} \mathrm{Ag}_{\mathrm{n}} \mathrm{Au}_{12-\mathrm{n}}(\mathrm{n}=0-12)$ kompozisyon sistemi için $\mathrm{Cu}, \mathrm{Ag}$ ve $\mathrm{Au}$ atomlarının Gupta düzeyinde ve DFT düzeyinde en kararlı nanoalaşımlara yerleşme eğilimlerinin benzer olduğu görülmektedir.

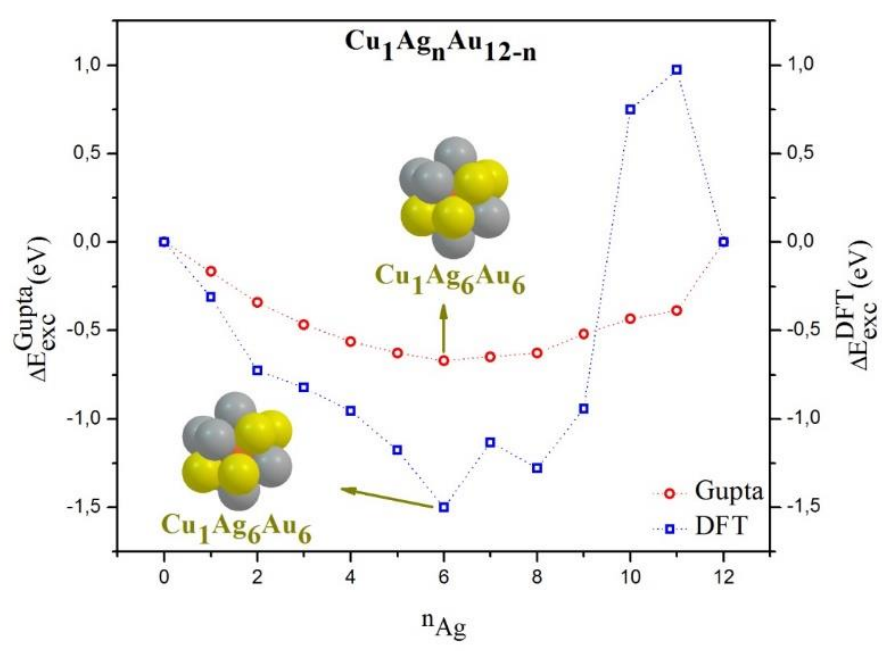

Şekil 4. $\mathrm{Cu}_{1} A g_{n} A u_{12-n}(n=0-12)$ nanoalaşımlarının Gupta ve DFT düzeyinde karışma enerjilerinin Ag sayısına göre değişimi.

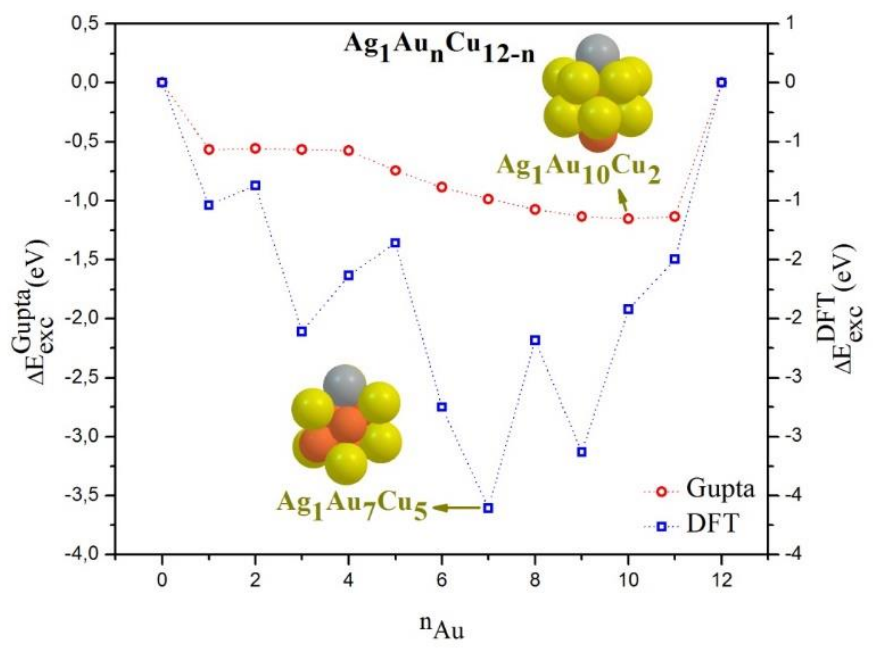

Şekil 5. $\mathrm{Ag}_{1} A u_{n} \mathrm{Cu}_{12-n}(n=0-12)$ nanoalaşımlarının Gupta ve DFT düzeyinde karışma enerjilerinin Au sayısına göre değişimi.

$\mathrm{Au}$ atomlarının sayısının (n) bir fonksiyonu olarak $\mathrm{Ag}_{1} \mathrm{Au}_{\mathrm{n}} \mathrm{Cu}_{12-\mathrm{n}}(\mathrm{n}=0-12)$ nanoalaşımlarının karışma enerjisi grafiği Şekil 5'te verilmiştir. Şekil 5'e göre, $\mathrm{Ag}_{1} \mathrm{Au}_{\mathrm{n}} \mathrm{Cu}_{12-\mathrm{n}}(\mathrm{n}=0-12)$ nanoalaşımlarının Gupta düzeyinde en kararlı nanoalaşımı $\mathrm{Ag}_{1} \mathrm{Au}_{10} \mathrm{Cu}_{2}$, DFT düzeyinde en kararlı nanoalaşımı ise $\mathrm{Ag}_{1} \mathrm{Au}_{7} \mathrm{Cu}_{5}$ olarak elde edilmiştir. $\mathrm{Bu}$ sonuç $\mathrm{Ag}_{1} \mathrm{Au}_{\mathrm{n}} \mathrm{Cu}_{12-\mathrm{n}}(\mathrm{n}=0-12)$ kompozisyon sistemi için Gupta ve DFT düzeylerinin birbirleriyle uyumlu olmadığını göstermektedir. Gupta düzeyindeki en kararlı kompozisyon $\mathrm{Ag}_{1} \mathrm{Au}_{10} \mathrm{Cu}_{2}$ nanoalaşımı Ortak Komşu Analizine göre ikosahedron yapıda iken, DFT düzeyindeki en kararlı kompozisyon $\mathrm{Ag}_{1} \mathrm{Au}_{7} \mathrm{Cu}_{5}$ nanoalaşımı amorf bir yapıya sahiptir. Ayrıca, $\mathrm{Ag}_{1} \mathrm{Au}_{\mathrm{n}} \mathrm{Cu}_{12-\mathrm{n}}(\mathrm{n}=0-12)$ kompozisyon sistemi için hem Gupta düzeyinde hem de DFT düzeyinde $\mathrm{Cu}, \mathrm{Ag}$ 
ve Au atomlarının en kararlı nanoalaşıma yerleşme eğilimi benzerlik göstermektedir. Nanoalaşımların merkezine $\mathrm{Cu}$ atomu yerleşirken, yüzeyine $\mathrm{Ag}$ ve $\mathrm{Au}$ atomları yerleşmektedir.

$\mathrm{Ag}$ atomlarının sayısının (n) bir fonksiyonu olarak $\mathrm{Au}_{1} \mathrm{Ag}_{\mathrm{n}} \mathrm{Cu}_{12-\mathrm{n}}(\mathrm{n}=0-12)$ nanoalaşımlarının karışma enerjisi grafiği Şekil 6'da verilmiş̧ir. Şekil 6'a göre, $\mathrm{Au}_{1} \mathrm{Ag}_{\mathrm{n}} \mathrm{Cu}_{12-\mathrm{n}}(\mathrm{n}=0-12)$ nanoalaşımlarının Gupta düzeyinde en kararlı nanoalaşımı $\mathrm{Au}_{1} \mathrm{Ag}_{6} \mathrm{Cu}_{6}$, DFT düzeyinde en kararlı nanoalaşımı ise $\mathrm{Au}_{1} \mathrm{Ag}_{1} \mathrm{Cu}_{11}$ olarak elde edilmiştir. $\mathrm{Bu}$ sonuç $\mathrm{Au}_{1} \mathrm{Ag}_{\mathrm{n}} \mathrm{Cu}_{12-\mathrm{n}}(\mathrm{n}=0-12)$ kompozisyon sistemi için Gupta ve DFT düzeylerinin birbirleriyle uyumlu olmadığını göstermektedir. Gupta düzeyindeki en kararlı kompozisyon $\mathrm{Au}_{1} \mathrm{Ag}_{6} \mathrm{Cu}_{6}$ nanoalaşımı Ortak Komşu Analizine göre ikosahedron yapıda iken, DFT düzeyindeki en kararlı kompozisyon $\mathrm{Au}_{1} \mathrm{Ag}_{1} \mathrm{Cu}_{11}$ nanoalaşımı amorf bir yapıya sahiptir. Ayrıca, Gupta düzeyindeki $\mathrm{Au}_{1} \mathrm{Ag}_{6} \mathrm{Cu}_{6}$ nanoalaşımında $\mathrm{Ag}$ ve $\mathrm{Cu}$ atomları birbirleriyle karışarak yüzeye yerleşirken, merkeze ise $\mathrm{Au}$ atomu yerleşmektedir. DFT düzeyindeki $\mathrm{Au}_{1} \mathrm{Ag}_{1} \mathrm{Cu}_{11}$ nanoalaşımında ise merkeze $\mathrm{Cu}$ atomu yerleşirken, $\mathrm{Ag}$ ve $\mathrm{Au}$ atomları birbirinden ayrışarak yüzeye yerleşmektedir. $\mathrm{Au}_{1} \mathrm{Ag}_{\mathrm{n}} \mathrm{Cu}_{12-\mathrm{n}}(\mathrm{n}=0-$ 12) kompozisyon sistemi için $\mathrm{Cu}, \mathrm{Ag}$ ve $\mathrm{Au}$ atomlarının, Gupta düzeyinde ve DFT düzeyinde elde edilen en kararlı nanoalaşımlara yerleşme eğilimlerinin farklı olduğu görülmektedir.

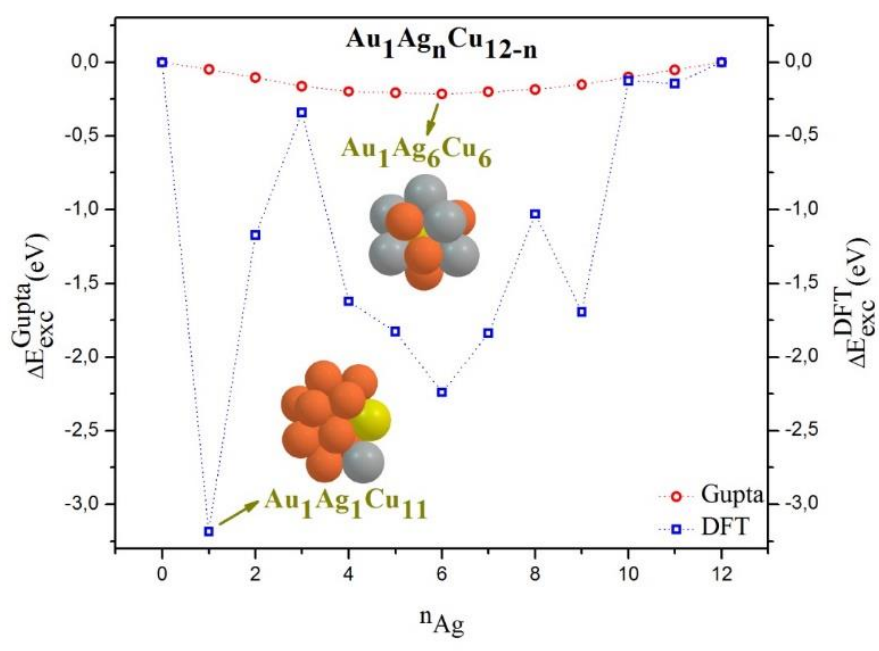

Şekil 6. $A u_{1} \mathrm{Ag}_{n} \mathrm{Cu}_{12-n}(n=0-12)$ nanoalaşımlarının Gupta ve DFT düzeyinde karışma enerjilerinin Ag saylsına göre değişimi.

$\mathrm{Cu}_{1} \mathrm{Ag}_{\mathrm{n}} \mathrm{Au}_{12-\mathrm{n}}(\mathrm{n}=0-12), \mathrm{Ag}_{1} \mathrm{Au}_{\mathrm{n}} \mathrm{Cu}_{12-\mathrm{n}}\left(\mathrm{n}=0\right.$-12) ve $\mathrm{Au}_{1} \mathrm{Ag}_{\mathrm{n}} \mathrm{Cu}_{12-\mathrm{n}}$ (n=0-12) kompozisyon sistemlerinde ikili nanoalaşımların karışma enerjisi değerleri hem Gupta hem de DFT düzeyinde $\mathrm{E}_{\text {exc }}=0$ olduğu görülmektedir. Bu sonuç üçlü nanoalaşımların hem Gupta düzeyinde hem de DFT düzeyinde genellikle ikili nanoalaşımlara göre daha kararlı olduğu anlamına gelmektedir. Yani üçlü nanoalaşımlar enerji analizine göre karışıma daha elverişlidir. Ayrıca, $\mathrm{Ag}_{1} \mathrm{Au}_{\mathrm{n}} \mathrm{Cu}_{12-\mathrm{n}}$ ( $\left.\mathrm{n}=0-12\right)$ ve $\mathrm{Au}_{1} \mathrm{Ag}_{\mathrm{n}} \mathrm{Cu}_{12-\mathrm{n}}(\mathrm{n}=0-12)$ kompozisyon sistemlerinde en kararlı nanoalaşımlar açısından Gupta ve DFT düzeyleri birbiriyle uyumsuz sonuçlar verirken, $\mathrm{Cu}_{1} \mathrm{Ag}_{\mathrm{n}} \mathrm{Au}_{12-\mathrm{n}}(\mathrm{n}=0-12)$ kompozisyon sisteminde birbiriyle uyumlu sonuç vermiştir.

$\mathrm{Cu}_{1} \mathrm{Ag}_{\mathrm{n}} \mathrm{Au}_{12-\mathrm{n}}(\mathrm{n}=0-12), \mathrm{Ag}_{1} \mathrm{Au}_{\mathrm{n}} \mathrm{Cu}_{12-\mathrm{n}}(\mathrm{n}=0-12)$ ve $\mathrm{Au}_{1} \mathrm{Ag}_{\mathrm{n}} \mathrm{Cu}_{12-\mathrm{n}}(\mathrm{n}=0-12)$ nanoalaşımlarında $\mathrm{Cu}, \mathrm{Ag}$ ve $\mathrm{Au}$ atomlarının yerleşme eğilimini daha iyi analiz edebilmek için nanoalaşımı oluşturan her bir tür atomun nanoalaşımın merkezinden ortalama uzaklığını ifade eden $\mathrm{R}_{\mathrm{A}}$ parametresi [20] değerleri elde edilmiştir.

$R_{A}=\frac{1}{n_{A}} \sum_{i=1}^{n_{A}} \sqrt{x_{i}^{2}+y_{i}^{2}+z_{i}^{2}}$ 
Burada, $n_{A}$ üçlü nanoalaşımlardaki her bir atom türünün atom sayısını, $x_{i}, y_{i}$ ve $z_{i}$ ise atomların konumunu göstermektedir. Büyük $\mathrm{R}$ değerleri atomların yüzeye yerleşme eğiliminde olduğunu gösterirken, küçük R değerleri ise atomların merkeze yerleşme eğiliminde olduğunu göstermektedir.

$\mathrm{Cu}_{1} \mathrm{Ag}_{\mathrm{n}} \mathrm{Au}_{12-\mathrm{n}}(\mathrm{n}=0-12), \mathrm{Ag}_{1} \mathrm{Au}_{\mathrm{n}} \mathrm{Cu}_{12-\mathrm{n}}(\mathrm{n}=0-12)$ ve $\mathrm{Au}_{1} \mathrm{Ag}_{\mathrm{n}} \mathrm{Cu}_{12-\mathrm{n}} \quad(\mathrm{n}=0-12)$ nanoalaşımlarının $\mathrm{R}$ parametrelerinin değişimi sırasıyla Şekil 7, Şekil 8 ve Şekil 9'da gösterilmiş̧ir. Şekil 7, Şekil 8 ve Şekil 9 incelediğinde üç kompozisyon sisteminde de $\mathrm{R}_{\mathrm{Ag}}$ değeri tüm nanoalaşımlarda $\mathrm{R}_{\mathrm{Au}}$ ve $\mathrm{R}_{\mathrm{Cu}}$ değerinden büyük olduğu görülmektedir. Bu sonuç $\mathrm{Ag}$ atomlarının tüm nanoalaşımlarda yüzeye yerleşmeyi tercih ettiğini göstermektedir. $\mathrm{Cu}_{1} \mathrm{Ag}_{\mathrm{n}} \mathrm{Au}_{12-\mathrm{n}} \quad(\mathrm{n}=0-12)$ nanoalaşımlarına ait $\mathrm{R}$ parametre değişimleri incelendiğinde $1 \mathrm{Cu}$ atomu $\mathrm{Cu}_{1} \mathrm{Ag}_{1} \mathrm{Au}_{11}$ nanoalaşımından $\mathrm{Cu}_{1} \mathrm{Ag}_{9} \mathrm{Au}_{3}$ nanoalaşımına kadar merkeze yerleşmeyi tercih ettiği için $R_{C u}$ değeri $R_{\text {Au }}$ değerinden küçüktür. $C_{1} A_{g_{10}} A_{u}$ ve ${ } u_{1} A_{11} A_{1}$ nanoalaşımlarında ise $A u$ atomları merkeze yerleştiği için $R_{C u}$ değeri $R_{A u}$ değerinden büyük olmaktadır.

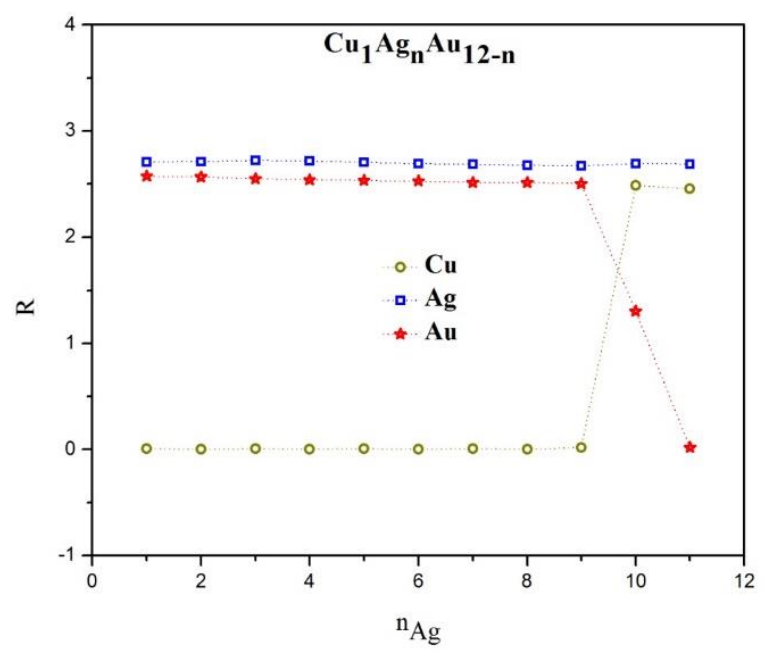

Şekil 7. $\mathrm{Cu}_{1} A g_{n} A u_{12-n}(n=0-12)$ nanoalaşımlarınin $R$ parametrelerinin değiş̧imi.

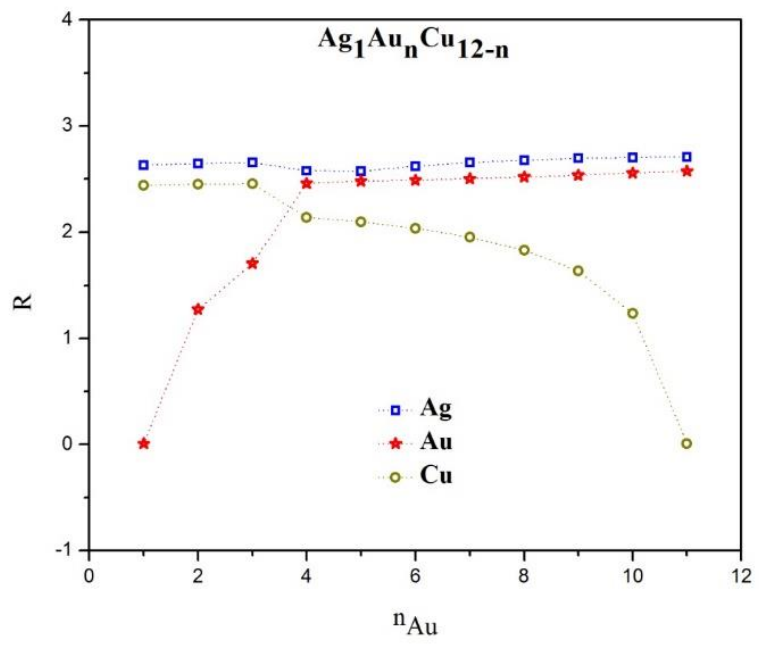

Şekil 8. $A g_{1} A u_{n} C u_{12-n}(n=0-12)$ nanoalaşımlarının $R$ parametrelerinin değiş̧imi.

$\mathrm{Ag}_{1} \mathrm{Au}_{\mathrm{n}} \mathrm{Cu}_{12-\mathrm{n}}$ ( $\mathrm{n}=0$-12) nanoalaşımlarına ait $\mathrm{R}$ parametre değişimleri incelendiğinde $\mathrm{Ag}_{1} \mathrm{Au}_{1} \mathrm{Cu}_{11}$ nanoalaşımından $\mathrm{Ag}_{1} \mathrm{Au}_{3} \mathrm{Cu}_{9}$ nanoalaşımına kadar $\mathrm{Au}$ atomları merkeze yerleşmeyi tercih ettiği için $\mathrm{R}_{\mathrm{Au}}$ 
değeri $\mathrm{R}_{\mathrm{Cu}}$ değerinden küçüktür. $\mathrm{Ag}_{1} \mathrm{Au}_{4} \mathrm{Cu}_{8}$ nanoalaşımından $\mathrm{Ag}_{1} \mathrm{Au}_{11} \mathrm{Cu}_{1}$ nanoalaşımına kadar $\mathrm{Cu}$ atomları merkeze yerleşmeyi tercih ettikleri için $\mathrm{R}_{\mathrm{Cu}}$ değeri, azalan $\mathrm{Cu}$ atomu sayısı ile azalmaktadır. $\mathrm{Au}_{1} \mathrm{Ag}_{\mathrm{n}} \mathrm{Cu}_{12-\mathrm{n}}(\mathrm{n}=0-12)$ nanoalaşımlarına ait $\mathrm{R}$ parametre değişimleri incelendiğinde ise $\mathrm{Au}$ atomları tüm nanoalaşımlarda merkeze yerleşmeyi tercih ettiği için $R_{A u}$ değeri $R_{C u}$ değerinden küçüktür. $R_{C u}$ değerinin, $R_{A u}$ değerine göre $R_{A g}$ değerine yakın olması ise $\mathrm{Cu}$ atomlarının $\mathrm{Ag}$ atomları ile birlikte yüzeye yerleşme eğilimi gösterdiğini ifade etmektedir. Sonuç olarak, $\mathrm{Cu}_{1} \mathrm{Ag}_{\mathrm{n}} \mathrm{Au}_{12-\mathrm{n}}(\mathrm{n}=0-12)$ ve $\mathrm{Ag}_{1} \mathrm{Au}_{\mathrm{n}} \mathrm{Cu}_{12-\mathrm{n}}(\mathrm{n}=0-12)$ kompozisyon sistemlerinde atomların merkeze veya yüzeye yerleşme eğilimi kompozisyona göre az da olsa değişiklik gösterirken, $\mathrm{Au}_{1} \mathrm{Ag}_{\mathrm{n}} \mathrm{Cu}_{12-\mathrm{n}}(\mathrm{n}=0-12)$ kompozisyon sisteminde atomların merkeze veya yüzeye yerleşme eğilimi kompozisyona göre değişmemektedir.

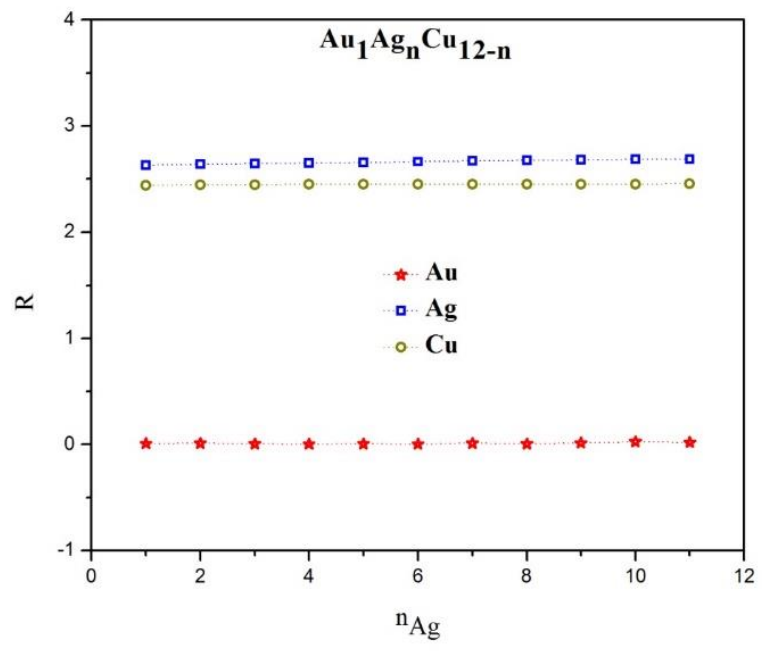

Şekil 9. $A u_{1} A g_{n} C u_{12-n}(n=0-12)$ nanoalaşımlarının $R$ parametrelerinin değişimi

\section{IV.SONUC}

13 atomlu $\mathrm{Cu}-\mathrm{Ag}-\mathrm{Au}$ nanoalaşımlarının yapısal özellikleri $\mathrm{Cu}_{1} \mathrm{Ag}_{\mathrm{n}} \mathrm{Au}_{12-\mathrm{n}}(\mathrm{n}=0-12), \mathrm{Ag}_{1} \mathrm{Au}_{\mathrm{n}} \mathrm{Cu}_{12-\mathrm{n}}(\mathrm{n}=0-$ 12) ve $\mathrm{Au}_{1} \mathrm{Ag}_{\mathrm{n}} \mathrm{Cu}_{12-\mathrm{n}}(\mathrm{n}=0-12)$ olacak şekilde oluşturulan üç farklı kompozisyon sisteminde Gupta ve DFT düzeylerinde incelenmiştir. Gupta düzeyinde $\mathrm{Cu}, \mathrm{Ag}$, $\mathrm{Au}$ atomlarının yerleşme eğilimleri $\mathrm{Cu}_{1} \mathrm{Ag}_{\mathrm{n}} \mathrm{Au}_{12-\mathrm{n}}(\mathrm{n}=0-12)$ ve $\mathrm{Ag}_{1} \mathrm{Au}_{\mathrm{n}} \mathrm{Cu}_{12-\mathrm{n}}(\mathrm{n}=0-12)$ kompozisyon sistemlerinde benzerlik gösterirken, $\mathrm{Au}_{1} \mathrm{Ag}_{\mathrm{n}} \mathrm{Cu}_{12-\mathrm{n}}(\mathrm{n}=0-12)$ kompozisyon sisteminde ise farklı olmaktadır. Ag atomlarının tüm kompozisyon sistemlerinde nanoalaşımın yüzeyine yerleşmeyi tercih ettiği görülmüştür. $\mathrm{Cu}$ ve $\mathrm{Au}$ atomlarının merkeze veya yüzeye yerleşme eğilimlerinin ise kompozisyon sistemine göre değiştiği görülmüştür. Gupta ve DFT düzeylerindeki karışma enerjisi analizlerine göre elde edilen en kararlı nanoalaşımlar açısından $\mathrm{Ag}_{1} \mathrm{Au}_{\mathrm{n}} \mathrm{Cu}_{12-\mathrm{n}}(\mathrm{n}=0-12)$ ve $\mathrm{Au}_{1} \mathrm{Ag}_{\mathrm{n}} \mathrm{Cu}_{12-\mathrm{n}}(\mathrm{n}=0-12)$ kompozisyon sistemlerinde Gupta ve DFT düzeyleri birbiriyle uyumsuz sonuçlar verirken, $\mathrm{Cu}_{1} \mathrm{Ag}_{\mathrm{n}} \mathrm{Au}_{12-\mathrm{n}}(\mathrm{n}=0-12)$ kompozisyon sisteminde birbiriyle uyumlu sonuç verdiği görülmüştür. DFT düzeyinde $\mathrm{Cu}_{1} \mathrm{Ag}_{\mathrm{n}} \mathrm{Au}_{12-\mathrm{n}}(\mathrm{n}=0-12)$ kompozisyon sisteminde en kararlı kimyasal düzene sahip nanoalaşım ikosahedron geometriye sahipken, $\mathrm{Ag}_{1} \mathrm{Au}_{\mathrm{n}} \mathrm{Cu}_{12}$ n $(\mathrm{n}=0-12)$ ve $\mathrm{Au}_{1} \mathrm{Ag}_{\mathrm{n}} \mathrm{Cu}_{12-\mathrm{n}}(\mathrm{n}=0-12)$ kompozisyon sistemlerinde amorf yapiya sahip olduğu görülmüştür. Sonuç olarak, $\mathrm{Cu}-\mathrm{Ag}-\mathrm{Au}$ nanoalaşımlarının yapısal özelliklerinin kompozisyon sistemine ve uygulanan yönteme göre değişiklik gösterdiği elde edilmiştir.

TEȘEKKÜR: $\mathrm{Bu}$ çalışmada gerçekleştirilen lokal optimizasyonlar Zonguldak Bülent Ecevit Üniversitesi Bilimsel Araştırma Projeleri tarafından desteklenen bilgisayar olanakları kullanarak yapılmıştır (Proje no: 2016-22794455-01, 2016-22794455-02). DFT hesaplamaları ise, TÜBİTAK-ULAKBİM TRUBA kaynaklarında gerçekleştirilmiştir. 


\section{KAYNAKLAR}

[1] A. K. Garip, H. Arslan, D. Rapetti, R. Ferrando, "A DFT study of chemical ordering and oxygen adsorption in AuPtPd ternary nanoalloys," Materials Today Communications, vol. 25, pp. 101545, 2020.

[2] X. Wu, G. Wu, Y. Chen, Y. Qiao, "Structural optimization of Cu-Ag-Au trimetallic clusters by adaptive immune optimization algorithm," The Journal of Physical Chemistry A, vol. 115, no. 46, pp. 13316-13323, 2011.

[3] S. Taran, H. Arslan, "Stability and magnetic behaviour of 19-,23- and 26- atom trimetallic PtNi-Ag nanoalloys," Molecular Physics, vol. 118, no. 23, pp. e1818859, 2020.

[4] S. A. C. Carabineiro, "Special Issue coinage metal (Copper, Silver, and Gold) catalysis," Molecules, vol. 21, no. 6, pp. 746, 2016.

[5] Y. Hashimoto, G. Seniutinas, A, Balcytis, S. Juodkazis, Y. Nishijima, "Au-Ag-Cu nano-alloys tailoring of permittivity," Scientific Reports, vol. 6, pp. 25010, 2016.

[6] C. Nwosu, "An electronegativity approach to catalytic performance," Journal of Technical Science and Technologies, vol. 1, no. 2, pp. 25-28, 2012.

[7] A. M. Echavarren, N. Jiao, V. Gevorgyan, "Coinage metals in organic synthesis," Chemical Society Reviews, vol. 45, no. 16, pp. 4445-4447, 2016.

[8] J. M. Conesa, M. V. Morales, C. Lopez-Olmos, I. Rodriguez-Ramos, A. Guerrero-Ruiz, "Comparative study of $\mathrm{Cu}, \mathrm{Ag}$ and $\mathrm{Ag}-\mathrm{Cu}$ catalysts over graphite in the ethanol dehydrogenation reaction: Catalytic activity, deactivation and regeneration," Applied Catalysis A, General, vol. 576, pp. 54-64, 2019.

[9] C. Syu, H. Yang, F. Hsu, J. Wang, "The chemical origin and catalytic activity of coinage metals: from oxidation to dehydrogenation," Physical Chemistry Chemical Physics, vol. 16, no. 16, pp. 74817490, 2014.

[10] K. Shin, D. H. Kim, H. M. Lee, "Catalytic characteristics of AgCu bimetallic nanoparticles in the oxygen reduction reaction," ChemSusChem, vol. 6, no. 6, pp. 1-7, 2013.

[11] N. Zhang, F. Chen, X. Wu, Q. Wang, A. Qaseem, Z. Xia, "The activity origin of core-shell and alloy $\mathrm{AgCu}$ bimetallic nanoparticles for the oxygen reduction reaction," Journal of Materials Chemistry A, vol. 5, no. 15, pp. 7043-7054, 2017.

[12] D. Cheng, X. Liu, D. Cao, W. Wang, S. Huang, "Surface segregation of $\mathrm{Ag}-\mathrm{Cu}-\mathrm{Au}$ trimetallic clusters," Nanotechnology, vol. 18, no. 47, pp. 475702, 2007.

[13] A. Rapallo, G. Rossi, R. Ferrando, A. Fortunelli, B. C. Curley, L. D. Lloyd, G. M. Tarbuck, R. L. Johnston, "Global optimization of bimetallic cluster structures. I. Size-mismatched Ag-Cu, Ag-Ni, and Au-Cu systems," The Journal of Chemical Physics, vol. 122, no. 19, pp. 194308, 2005.

[14] N. T. Wilson, R. L. Johnston, "A theoretical study of atom ordering in copper-gold nanoalloy clusters,” Journal of Materials Chemistry, vol. 12, pp. 2913-2922, 2002.

[15] X. Wu, W. Cai, X. Shao, "Optimization of bimetallic $\mathrm{Cu}-\mathrm{Au}$ and Ag-Au clusters by using a modified adaptive immune optimization algorithm," Journal of Computational Chemistry, vol. 30, no. 13, pp.1992-2000, 2009. 
[16] H. Yıldırım, H. Arslan, "CuAgAu üçlü nanoalaşımların optimizasyonu ve erime dinamiği," Balıkesir Üniversitesi Fen Bilimleri Enstitüsü Dergisi, c. 21, s. 1, ss. 336-351, 2019.

[17] H. Y1ldırım, H. Arslan, "Size and composition effect on structural properties and melting behaviors of $\mathrm{Cu}-\mathrm{Ag}-\mathrm{Au}$ ternary nanoalloys," International Journal of Modern Physics C, vol. 31, no. 6, pp. 2050078, 2020.

[18] J. Goh, J. Akola, R. Ferrando, "Geometric structure and chemical ordering of large AuCu clusters:A computational study," The Journal of Physical Chemistry C, vol. 121, no. 20, pp. 1080910816, 2017.

[19] Z. Jiang, K. Lee, S. Li, S. Chu, "Structures and charge distributions of cationic and neutral $\mathrm{Cu}_{\mathrm{n}-}$ ${ }_{1}$ Ag clusters (n=2-8)," Physical Review B, vol. 73, no.23, pp. 235423, 2006.

[20] S. Taran, A. K. Garip, H. Arslan, "Investigation of the chemical ordering and structural properties of the trimetallic (PtNi)@Ag nanoalloys," Journal of Cluster Science, vol. 32, pp. 199-208, 2021.

[21] S. Taran, "13 atomlu Cu-Au-Pt üçlü metal nanoalaşımların yapısal özellikleri," Düzce Üniversitesi Bilim ve Teknoloji Dergisi, c. 7, ss. 1204-1216, 2019.

[22] S. Taran, "Composition effect on melting behaviors of $\mathrm{Cu}-\mathrm{Au}-\mathrm{Pt}$ trimetallic nanoalloys," Computational and Theoretical Chemistry, vol. 1166, pp. 112576, 2019.

[23] S. Taran, "Alloying effect on the local atomic pressures of nanoclusters," Sakarya University Journal of Science, vol. 24, no. 3, pp. 501-510, 2020.

[24] R. Ferrando, "Structure and properties of nanoalloys," in Frontiers of Nanoscience, c.10, 1st. Ed. Elsevier, 2016, ss. 350. [Online]. https://www.elsevier.com.

[25] D. J. Wales, J. P. K. Doye, "Global optimization by basin-hopping and the lowest energy structures of lennard-jones clusters containing up to 110 Atoms," The Journal of Physical Chemistry A, vol. 101, no. 28, pp. 5111-5116, 1997.

[26] F. Cleri, V. Rosato, "Tight-binding potentials for transition metals and alloys," Physical Review $B$, vol. 48, no. 1, pp. 22-33, 1993.

[27] L. O. Paz-Borbon, R. L. Johnston, G. Barcaro, A. Fortunelli, "Structural motifs, mixing, and segregation effects in 38-atom binary clusters," The Journal of Chemical Physics, vol. 128, no. 13, pp. $134517,2008$.

[28] A. K. Garip, H. Arslan, "40 atomlu Pd-Co ikili metal atom topaklarının yapısal özelliklerinin incelenmesi," Karaelmas Fen ve Mühendislik Dergisi, c. 4, s. 2, ss. 38-45, 2014.

[29] A. K. Garip, "Kesilmiş oktahedron yapısına sahip $\operatorname{PdnPt}_{(6-n)} \mathrm{Au}_{32}$ nanoalaşımlarının erime dinamiği," Düzce Üniversitesi Bilim ve Teknoloji Dergisi, c. 8, ss. 1732-1745, 2020.

[30] P. Giannozzi, S. Baroni, N. Bonini, M. Calandra, R. Car, C. Cavazzoni, D. Ceresoli, G. L. Chiarotti, M. Cococcioni, I. Dabo, A. Dal Corso, S. De Gironcoli, S. Fabris, G. Fratesi, R. Gebauer, U. Gerstmann, C. Gougoussis, A. Kokalj, M. Lazzeri, L. Martin-Samos, N. Marzari, F. Mauri, R. Mazzarello, S. Paolini, A. Pasquarello, L. Paulatto, C. Sbraccia, S. Scandolo, G. Sclauzero, A. P. Seitsonen, A. Smogunov, P. Umari, R. M. Wentzcovitch, "QUANTUM ESPRESSO: a modular and open-source software project for quantum simulations of materials," Journal of Physics:Condensed Matter, vol. 21, no. 39, pp. 395502, 2009. 
[31] P. Giannozzi, O. Andreussi, T. Brumme, O. Bunau, M. B. Nardelli, M. Calandra, R. Car, C. Cavazzoni, D. Ceresoli, M. Cococcioni, N. Colonna, I. Carnimeo, A. Dal Corso, S. De Gironcoli, P. Delugas, R. A. DiStasio Jr., A. Ferretti, A. Floris, G. Fratesi, G. Fugallo, R. Gebauer, U. Gerstmann, F. Giustino, T. Gorni, J. Jia, M. Kawamura, H. Ko, A. Kokalj, E. Küçükbenli, M. Lazzeri, M. Marsili, N. Marzari, F. Mauri, N. L. Nguyen, H. Nguyen, A. De La Roza, L. Paulatto, S. Ponce, D. Rocca, R. Sabatini, B. Santra, M. Schlipf, A. P. Seitsonen, A. Smogunov, I. Timrov, T. Thonhauser, P. Umari, N. Vast, X. Wu, S. Baroni, "Advanced capabilities for materials modelling with Quantum ESPRESSO," Journal of Physics:Condensed Matter, vol. 29, no. 46, pp. 465901, 2017.

[32] D. Vanderbilt, "Soft self-consistent pseudopotentials in a generalized eigenvalue formalism," Physical Review B, vol. 41, no. 11, pp. 7892-7895, 1990.

[33] J. P. Perdew, K. Burke, M. Ernzerhof, "Generalized gradient approximation made simple," Physical Review Letters, vol. 77, no. 18, pp. 3865-3868, 1996.

[34] A. Stukowski, "Visualization and analysis of atomistic simulation data with OVITO-the Open Visualization Tool," Modelling and Simulation in Materials Science and Engineering, vol. 18, no.1, pp. $015012,2010$.

[35] A. Stukowski, "Structure identification methods for atomistic simulations of crystalline materials," Modelling and Simulation in Materials Science and Engineering, vol. 20, no. 4, pp. 045021, 2012.

[36] R. Ferrando, J. Jellinek, R. L. Johnston, "Nanoalloys: From Theory to Applications of Alloy Clusters and Nanoparticles," Chemical Reviews, vol.108, no. 3, pp. 845-910, 2008.

[37] S. Taran, A. K. Garip, H. Arslan, "A theoretical study on chemical ordering of 38-atom trimetallic Pd-Ag-Pt nanoalloys," Chinese Physics B, vol. 29, no. 7, 2020. 